\title{
AN X-RAY IMAGE OF THE LARGE MAGELLANIC CLOUD: DETECTION OF THE HOT INTERSTELLAR MEDIUM
}

\author{
D. J. Helfand \\ Columbia Astrophysics Laboratory \\ Columbia University \\ 538 West $120^{\text {th }}$ Street \\ New York, NY 10027
}

\begin{abstract}
We report the results of a comprehensive reanalysis of the X-ray imaging data for the Large Magellanic Cloud obtained with the Einstein Observatory. A map covering $37 \mathrm{deg}^{2}$ of the Cloud is presented. The total $0.15-3.5 \mathrm{keV}$ luminosity is $\sim 5 \times 10^{38} \mathrm{erg} \mathrm{s}^{-1}$, two thirds of which can be attributed to the 105 identified discrete emitters and the remainder of which arise from the coronal component of the interstellar medium at temperatures from $\sim 2$ to $\sim 10 \times 10^{6} \mathrm{~K}$. The energy balance for this hot ISM and the morphology of specific regions such as 30 Doradus and LMC-2 are briefly discussed.
\end{abstract}

\section{Introduction}

The notion that gas with a temperature of $\gtrsim 10^{6} \mathrm{~K}$ might exist in interstellar space originated over thirty years ago with Spitzer's classic paper on the galactic corona (Spitzer 1956). The fact that coronal gas is a major constituent of the interstellar medium (ISM) even in the plane of the Galaxy became apparent twenty years later with the pioneering detections of UV resonance absorption lines from highly ionized species such as OVI (e.g., Rogerson et al. 1973; Jenkins \& Meloy 1974, York 1974) and observations of the soft X-ray background (see McCammon et al. 1983 for a review). Theoretical work on the hot ISM began with papers by Cox and Smith (1974) and Castor et al. (1975). Arguments for a dominant coronal component were first advanced by McKee and Ostriker (1977); additional work on the issue has been pursued by Shapiro and Field (1976), Ikeuchi (1987), and Norman and Ikeuchi (1989).

Despite considerable work over the last decade, however, the filling factor of the hot component of the ISM is still very uncertain. The principal difficulty, of course, is that our observing platform is embedded in the medium for which we are attempting to adduce a global model, and it is clear that an external perspective would be enormously helpful. While we have been unable to effect such an observation, we can provide such a perspective on our nearest neighbor galaxy, the Large Magellanic Cloud (LMC). The detection and mapping of the coronal gas in that galaxy reported here provides an important test of global ISM models. Combining these results with data from other wavelength regimes will allow us to examine the energy budget, structure, and evolution of the medium in seeking to delineate the central role it plays in mediating the star formation rate and in reflecting the evolution of the galaxy as a whole.

The LMC was the subject of an extensive survey conducted with the instruments onboard the Einstein Observatory between 1979 and 1981. Based on data collected with the Observatory's Imaging Proportional Counter (IPC) and High Resolution Imager (HRI), Long et al. (1981; hereafter LHG) published a list of 97 discrete X-ray sources detected in the direction of the Cloud, providing 
the first X-ray census of an external galaxy to a limiting luminosity of $\sim 10^{35} \mathrm{erg} \mathrm{s}^{-1}$ in the $0.2-4.5$ $\mathrm{keV}$ band. Followup observations carried out by a number of groups (Mathewson et al. 1983; 84; 85; Cowley et al. 1984; Pakull 1984) identified optical and/or radio counterparts for roughly half of these X-ray emitters including supernova remnants, X-ray binary systems, and foreground (stars) and background (AGN) interlopers. Additional X-ray observations of the Cloud reported in the last decade include an EXOSAT pointing which found an additional X-ray source EXO 053109-6609.2 (a recurrent transient identified with a Be-type binary system) in the vicinity of LMC X-4 (Pakull et al. 1985; Pietsch et al. 1989); spectroscopic observations of the brightest SNRs using the Einstein instruments (Clark et al.; Hughes 1987), and a map of the apparently diffuse emission in the vicinity of the Cloud derived from HEAO-1 A-2 LED database (Singh et al. 1987).

With the exception of an image of the region surrounding the site of SN1987A presented by Harnden and Seward (1987 - see also Wang et al. 1989), all analysis of the Einstein imaging data to date has been performed by treating each pointing of from $10^{2.5}-10^{4.5} \mathrm{~s}$ as a separate observation. Over the past few years, however, we have developed at Columbia University new algorithms for merging adjacent and/or overlapping observations obtained with the IPC; in addition to the obvious benefit of improved sensitivity, these algorithms have allowed us to calibrate out a number of instrumental artifacts and to measure the contamination in IPC images from solar scattered X-rays and from cosmic ray events in the detector. As a result, we are now in a position to construct a global map of the LMC with optimum sensitivity to discrete sources and with an absolute photometric accuracy for diffuse emission of $\sim 7 \times 10^{-7} \mathrm{ph} \mathrm{cm}^{-2} \operatorname{arcmin}^{-2} \mathrm{~s}^{-1}$ in the $0.16-3.5 \mathrm{keV}$ band.

We have recently completed a comprehensive reanalysis of all X-ray imaging observations of the Cloud conducted with the Einstein IPC. The results are reported in a series of four papers which have been submitted to The Astrophysical Journal, and in the Ph.D. dissertation of Dr. Qingde Wang. The papers are, respectively: "The Detection of X-rays from the Hot Interstellar Medium of the LMC" (Wang et al. 1990), "An X-ray Image of the Violent ISM in 30 Doradus" (Wang and Helfand 1990a); "LMC-2 as the Blowout of a Hot Supergiant Bubble" (Wang and Helfand 1990b); and "The Detection of X-rays from OB Associations in the LMC" (Wang and Helfand 1990c). The latter paper is summarized by Wang and Helfand (1990); work on the same subject is also reported here by Chu (1990) and in the soon-to-be-published Astrophysical Journal paper by Chu and MacLow (1990). We refer the reader to those works for a discussion of the detection of hot gas from the direction of over two dozen LMC associations and its interpretation as arising in supernova-heated wind-blown bubbles. Below, we briefly summarize the remainder of our work on X-rays from the Large Cloud.

\section{Discrete X-ray Sources}

A total of 105 discrete X-ray sources, defined as enhancements $\leq 3^{\prime}(50 \mathrm{pc})$ in diameter which exceed the local background by $4 \sigma$, have been detected in the $37 \mathrm{deg}^{2}$ region covered by the survey. Twenty-eight of these are supernova remnants also seen in the radio and/or optical bands, nine are $\mathrm{X}$-ray binary sources, and 20 are associated with $\mathrm{OB}$ associations or other evidence of star formation activity. There are also 13 foreground Galactic stars and three identified background AGN in the image. An analysis of the expected contamination from background interlopers including the effects of absorption by LMC gas indicates that most if not all of the remaining 32 sources are also AGN. The status of the optical identification program for discrete X-ray sources in the Cloud with an emphasis on studies of the X-ray binary population may be found in the report of Hutchings (1990).

\section{Global Diffuse Emission}

Figure 1 displays an X-ray image of the Large Cloud after removal of all discrete emitters. Details of the map's construction including the removal of contamination from solar-scattered Xrays and cosmic ray particles, flat-fielding, mosaicing, smoothing, and source excision may be found 


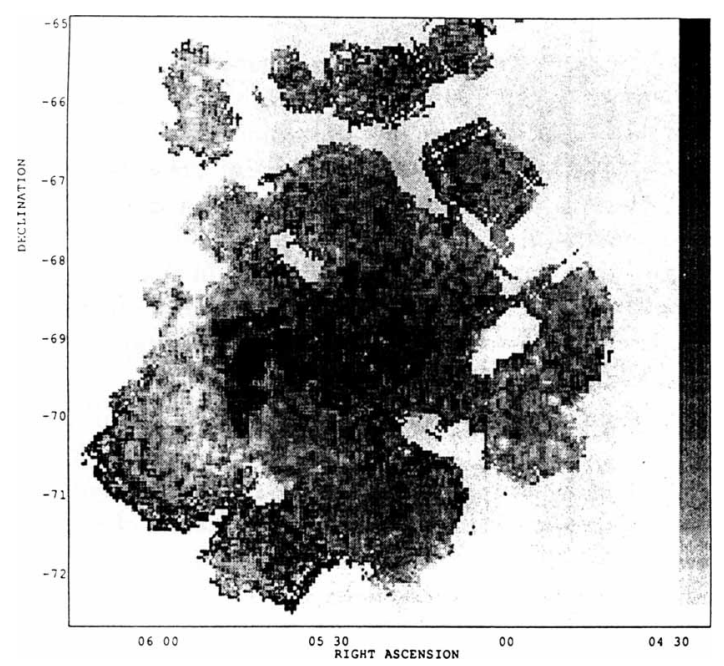

Figure 1. A greyscale representation of the diffuse X-ray emission in the direction of the LMC.

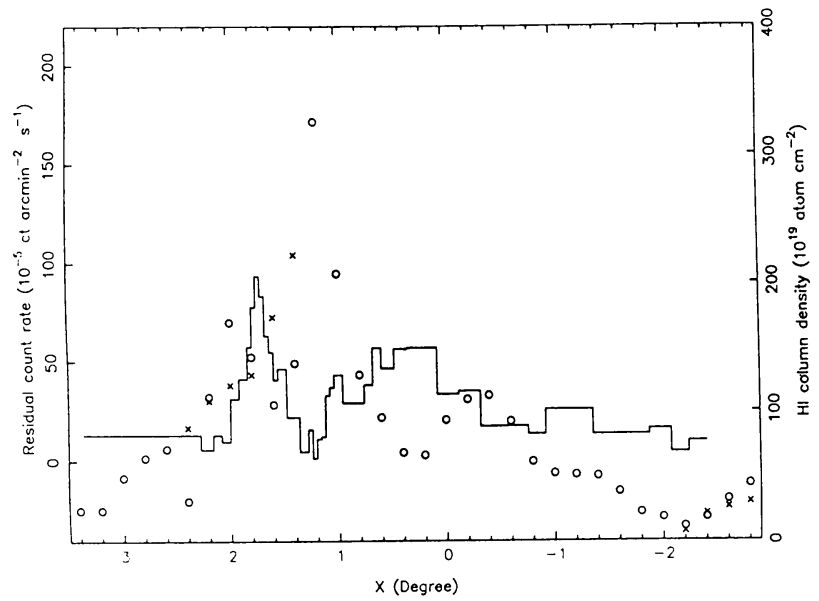

Figure 2. A plot of the surface brightness for the diffuse X-ray emission (solid curve) and the neutral hydrogen column density (open circles and x's curve) in a slice 12' wide through the LMC at declination $-66^{\circ} 59^{\prime}$. The X-axis in units of degrees refers to a coordinate adopted by Rohlfs et al. (1984) and centred on the optical centre of the LMC bar. The horizontal lines of varying lengths indicate the regions over which the X-ray surface brightness was determined (so that each has an equivalent statistical uncertainty). The two symbols for the HI distribution represent the two velocity components seen in some directions toward the LMC ( 0 = lower velocity; $x=$ higher velocity). The anticorrelation of the $\mathrm{X}$-rays and $\mathrm{HI}$ on scales of 0.5 to $1 \mathrm{kpc}\left(30^{\prime}\right.$ to $\left.1^{\circ}\right)$ is apparent, although on a global scale, the eastern half of the Cloud has both a higher HI column density and a higher mean X-ray emissivity. 
in Wang et al. (1990) and in Wu et al. (1990). The mean surface brightness over the entire region is $4.1 \times 10^{-4} \mathrm{ct}_{\text {arcmin }}^{-2} \mathrm{~s}^{-1}, \sim 65 \%$ higher than the flux from average high galactic latitude fields in the same energy band. Adopting a 50 -kpc distance to the LMC, the integrate luminosity of this diffuse emission is $\sim 2 \times 10^{38} \mathrm{erg} \mathrm{s}^{-1}$, or over one-third of the total X-ray luminosity of the galaxy. The surface brightness varies by more than an order of magnitude over the face of the galaxy, from a minimum near $\mathrm{RA}=05^{\mathrm{h}} 50^{\mathrm{m}}$, Dec $=070^{\circ} 20^{\prime}$ which is significantly below the mean diffuse X-ray background from other regions of the sky, to a maximum only one degree away near the 30 Dor complex. The low point corresponds to the peak of the neutral gas column density in the Cloud and represents the first detection of a shadow cast on the cosmic background radiation by absorbing gas along the line of sight, while the peak emissivity is part of the extensive and complex X-ray emission associated with the hot component of the Cloud's interstellar medium.

Spectral analysis of the diffuse emission reveals that the X-rays arise from optically thin gas at a temperature which ranges from $\mathrm{kT} \sim 0.3 \mathrm{keV}$ in the western edge of the Cloud to over $1 \mathrm{keV}$ in the vicinity of 30 Doradus. Comparison of the X-ray surface brightness with the neutral hydrogen column density, an example of which is shown in Fig. 2, shows an anticorrelation on scales of $\sim 1 \mathrm{kpc}$, consistent with a picture in which X-ray bright areas represent regions in which large cavities filled with coronal gas have been excavated in the neutral interstellar medium by the combined action of stellar winds and supernovae arising in spatially correlated massive star formation complexes (McCray and Kafatos 1987).

Scaling to a mean value of $T=4 \times 10^{6} \mathrm{~K}$ and a surface brightness $S=10^{-4} \mathrm{ct} \operatorname{arcmin}^{-2} \mathrm{~s}^{-1}$, we can calculate the emission measure $n_{e}^{2} h$ of the hot gas:

$$
\begin{gathered}
n_{e}^{2} h \approx\left(5 \times 10^{-2} \mathrm{~cm}^{-6} \mathrm{pc}\right)\left(\frac{T}{4 \times 10^{6} \mathrm{k}}\right)^{0.7}\left(\frac{\eta}{4 \times 10^{37} \mathrm{erg} \mathrm{ct}^{-1}}\right) \\
\left(\frac{S}{10^{-4} \mathrm{ct} \operatorname{arcmin}^{-2} \mathrm{~s}^{-1}}\right)\left(\frac{z}{0.3}\right)^{-1}
\end{gathered}
$$

where $z$ is the metallicity of the gas relative to solar abundances and $\eta$ is a factor which includes the conversion of IPC ct s${ }^{-1}$ to erg s$~^{-1}$ for an emitter at the distance of the LMC including the emission from the thermal plasma outside the $0.16-3.5 \mathrm{keV}$ band; the uncertainty in this parameter owing to the range of temperatures and absorption involved is at least a factor of 2 . The emission measure is $\gtrsim 5$ times that characteristic of the Milky Way's ISM. These observations, then, reveal a higher pressure ISM for the LMC, particularly in the east, demonstrating the feedback mechanism in which a high pressure medium accelerates star formation activity which, in turn, leads to a higher pressure medium.

Examination of the energy budget of the hot medium is also instructive in this regard. While the undetermined relative locations along the line of sight for the emitting and absorbing gas renders uncertain any estimate of the cooliing rate for the hot ISM, the X-rays we directly observe provide an unambiguous lower limit of $>2 \times 10^{38} \mathrm{erg} \mathrm{s}^{-1}$; emission at longer wavelengths and from gas at lower temperatures will most likely raise this value to $\sim 10^{39} \mathrm{erg} \mathrm{s}^{-1}$. The total thermal energy in the hot component, $E_{T}$, over the $37 \mathrm{deg}^{2}$ field may be calculated from Eq. (1):

$$
\begin{gathered}
E_{T} \approx\left(6 \times 10^{54} \mathrm{ergs}\right)\left(\frac{T}{4 \times 10^{6} \mathrm{~K}}\right)^{1.35}\left(\frac{S}{10^{-4}}\right)^{0.5}\left(\frac{z}{0.3}\right)^{-0.5} \\
\left(\frac{\eta}{4 \times 10^{37} \mathrm{erg} \mathrm{ct}^{-1}}\right)^{0.5}\left(\frac{h}{1 \mathrm{kpc}}\right)^{0.5}
\end{gathered}
$$

where $h$ is the thickness of the gas layer. Combining equations (1) and (2) leads to a cooling time for the hot gas of $\sim 10^{8} \mathrm{yr}$. 
If we assume the gas is predominantly heated by the combined action of stellar winds and supernovae, we can estimate the heating rate as

$$
\sim\left(10^{48} \mathrm{erg} \mathrm{yr}^{-1}\right)\left(\frac{E_{\mathrm{SN}}}{10^{51} \mathrm{ergs}}\right)\left(\frac{t_{\mathrm{SN}}}{10^{3} \mathrm{yr}}\right)^{-1},
$$

where $E_{\mathrm{SN}}$ is the energy per supernova which couples to the ISM, and $t_{\mathrm{SN}}$ is the time between supernovae. The heating timescale, then, is $\leqslant 10^{7} \mathrm{yrs}$, an order of magnitude less than the cooling time. It appears that a substantial fraction of the energy which massive stars impart to their surrounding medium leaves the galaxy in a form other than thermal radiaiton from gas at a temperature of $10^{6}-10^{7} \mathrm{~K}$. This is consistent with the conclusion of Reynolds (1990) for the Milky Way based on the ionization of gas at high latitudes. Bubbles of hot gas generated by correlated massive star formation may break out of the disk of the galaxy, carrying away substnatial amounts of energy, either for deposit in a hot halo or in the form of a galactic wind (McCray \& Kafatos 1987). Further analysis of the diffuse emission in the vicinity of bubbles and $\mathrm{OB}$ associations as well as in the shadowing region, along with future observations of the UV absorption along lines of sight through the Cloud using the AGN discovered from our survey will be helpful in assessing this suggestion.

\section{The 30 Doradus Complex}

As the most luminous HII region in the Local Group, the 30 Doradus Nebula provides a striking example of the complex interrelationship between massive star formation and the structure of the interstellar medium. The X-ray observations presented here provide strong evidence that the dominant interstellar component in such starburst regions consists of X-ray emitting gas at a temperature of $\sim 10^{6.5} \mathrm{~K}$. By applying a new maximum entropy deconvolution algorithm we have developed (Wang and Helfand 1990a) we find a total luminosity in the $0.16-3.5 \mathrm{keV}$ band of $2 \times 10^{37} \mathrm{erg} \mathrm{s}^{-1}$ from a circular region within $160 \mathrm{pc}$ of R136, with more than half of this emission diffuse at $1^{\prime}$ resolution. There is a strong correlation between the X-ray, radio and optical morphologies in the region, with $\mathrm{X}$-ray emitting bubbles filling cavities surrounded by $\mathrm{H} \alpha$ shells and coextensive diffuse X-ray and radio continuum emission from throughout the region. An illustration of the $\mathrm{X}$-ray/radio correlation is shown in Figure 3 where the $845 \mathrm{MHz}$ contours of Mills and Turtle (1984) are superposed on a greyscale representation of the $\mathrm{X}$-ray emission. The temperature of the hot gas producing the $\mathrm{X}$-rays is $\sim 5 \times 10^{6} \mathrm{~K}$, implying a total thermal energy of $\sim 10^{52.5} \mathrm{ergs}$. From an analysis of the $\mathrm{X}$-ray emission, taken in conjunction with information from optical and radio wavelengths, we find consistency with a general scenario in which stellar wind- and supernova-driven bubbles carve up the ISM into compressed $\mathrm{H} \alpha$ filaments surrounding cavities filled with coronal gas, which dominates the space in the Nebula.

\section{LMC 2 - Blowout of a Supergiant Bubble}

To the southeast of the 30 Dor complex lies LMC-2 a supergiant shell of $\mathrm{H} \alpha$-emitting filaments first identified by Meaburn $(1979 ; 1980)$. We have found X-ray emission from a $0.2 \mathrm{kpc}^{2}$ region within the shell which has an integrated luminosity of $\sim 10^{37} \mathrm{erg} \mathrm{s}^{-1}$ and a temperature $\mathrm{kT} \sim 0.5$ $\mathrm{keV}$. The X-ray emitting region correlates well with a cavity in the cold ISM marked by the HI and IRAS far-infrared maps (Schwering 1988; Dwek 1988 priv. comm.), but is considerably smaller than the area bounded by the optical filaments. Taking into account the available kinematic data from $\mathrm{HI}$ and optical observations, we can model this region as the breakout from the plane of the LMC of a supergiant bubble formed from the accumulated effects of stellar winds and supernovae over the past $\gtrsim 10^{7} \mathrm{yr}$. Figure 4 illustrates how, when viewed from an angle $\sim 20^{\circ}$ to the plane of the galaxy, the X-rays will appear to fill only part of the region surrounded by optical filaments. The venting of this hot gas to the halo of the LMC (or beyond) may play an important role in the energy balance of the galaxy's interstellar medium. 


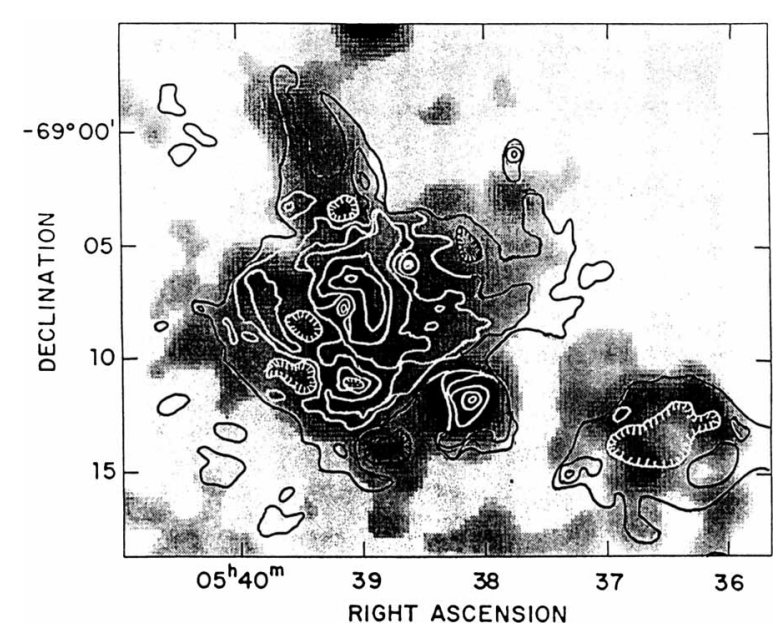

Figure 3. A greyscale version of the central region of X-ray emission from the 30-Dor complex overlain with contours from the $845 \mathrm{MHz}$ radio continuum map of Mills and Turtle (1984).

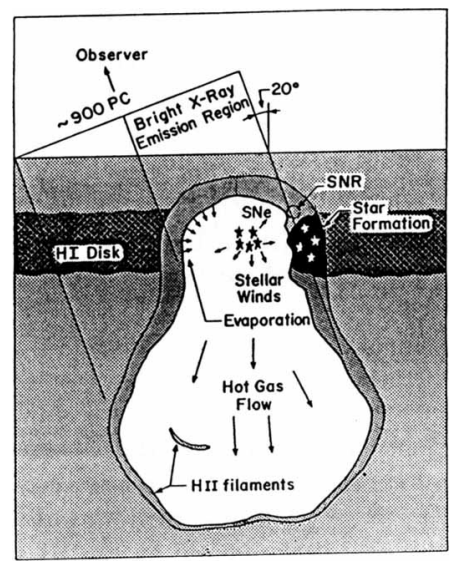

Figure 4. An illustration of the geometry of the supergiant shell (SGS) blow out. LMC 2 is viewed at an inclination angle of $\sim 20^{\circ}$ and is venting hot gas out of the far side of the dense ISM disk of the LMC. Bright X-ray emission comes from the direction of the cold ISM cavity, where SNe and stellar winds evaporate the ISM. The hot gas flows out of the disk and drives the expansion of the SGS. The rimbrightened optical filaments give a projection of the SGS which has an asymmetric shape and a larger dimension than that of the HI cavity or the bright X-ray emission region. The edge-on view of the right side of the SGS may partially explain the bright ridge observed in several wavelength bands. 


\section{Summary}

Using the Einstein Observatory X-ray data we have produced the first image of the hot interstellar medium in an external galaxy. These data illustrate the complex interaction and feedback mechanisms governing star formation and the structure of interstellar gas, and establish important constraints on global models for the ISM. More sensitive soft X-ray images of both Magellanic Clouds should soon be available from ROSAT, providing further information on the coronal gas and confirming the utility of the Clouds as a testbed for our ideas about the structure and evolution of galaxies.

\section{Acknowledgements}

This work, supported by NASA under grants NAG8-665 and NAG8-497 comprises the doctoral disssertation research for Qingde Wang. Dr. Xiaoyi Wu and Dr. Thomas Hamilton were collaborators on several aspects of the project. This paper is Contribution number 431 of the Columbia Astrophysics Laboratory.

\section{References}

Castor, J., McCray, R., Weaver, R. 1975, Ap. J. 200, L107.

Chu, Y.-H., and Mac Low, M.-M 1990, Ap.J. (in press).

Chu, Y.-H 1990, (these proceedings).

Clark, D.H., Tuohy, I.R., Long, K.S., Szymkowiak, A.E., Dopita, M.A., Mathewson, D.S., and Culhane, J.L. 1982, Ap.J., 255, 440.

Cowley, A.P., Crampton, D., Hutchings, J.B., Helfand, D.J., Hamilton, T.T., Thorstensen, J.R., and Charles, P.A. 1984, Ap.J., 286, 196.

Cox, D.P., and Smith, B.W. 1974, Ap.J. (Letters), 189, L105.

Harnden, F.R., and Seward, F.D. 1987, in Proceedings of the Fourth George Mason Astrophysics Workshop, eds. Kafatos and Michalitsianos, p. 408.

Hughes, J.P. 1987, Ap.J., 314, 103.

Hutchings, J. 1990, (these proceedings).

Ikeuchi, S. 1987, in Starbursts and Galaxy Evolution, eds. T.X. Thuan, T. Montmerle and J.T. Thanh Van, p. 27.

Jenkins, E.B., Meloy, D.A. 1974, Ap. J. 193, L121.

Long, K.S., Helfand, D.J., and Grabelsky, D.A. 1981, Ap.J., 248, 925.

Mathewson, D.S., Ford, V.L., Dopita, M.A., Tuohy, I.R., Long, K.S., and Helfand, D.J. 1983, Ap.J. Suppl., 51, 345 .

Mathewson, D.S., Ford, V.L., Dopita, M.A., Tuohy, I.R., Mills, B.Y., and Turtle, A.J. 1984, Ap.J. Suppl., 55, 189.

Mathewson, D.S., Ford, V.L., Tuohy, I.R., Mills, B.Y., Turtle, A.J., and Helfand, D.J. 1985, Ap.J. Suppl., 58, 197.

McCammon, D., Burrows, D.N., Sanders, W.T., and Kraushaar, W.L. 1983, Ap.J., 269, 107.

McKee, C.F., and Ostriker, J.P. 1977, Ap.J., 218, 148.

Meaburn, J. 1979, Astr. Ap., 75, 127.

Meaburn, J. 1980, M.N.R.A.S., 192, 365.

McCray, R., and Kafatos, M. 1987, Ap.J., 317, 190.

Mills, B.Y., and Turtle, A.J. 1984, in IAU Symposium 108, Structure and Evolution of the Magellanic Cloud, eds. S. van den Bergh and K. de Boer (Dordrecht: Reidel), p. 283.

Norman, C.A., Ikeuchi S. 1989, Ap. J. 345, 372.

Pakull, M.W. 1984, in X-ray and UV Emission from Active Galactic Nuclei, eds. W. Brinkmann and J. Trümps, p. 28. 
Pakull, M.W. et al. 1985, Space Sci. Rev., 40, 379.

Pietsch, W., Denneil, K., and Rosso, C. 1989, in $23^{r d}$ ESLAB Symposium on X-ray Astronomy, (in press).

Reynolds, R.J. 1990, Ap.J. (Letters), 349, L17.

Rogerson, J.B., York, D.G., Drake, J.F., Jenkins, E.B., Morton, D.C., and Spitzer, L. 1973, Ap.J. (Letters), 181, L110.

Rohlfs, K., Kreitschmann, J., Siegman, B.C., and Feitzinger, J.V. 1984, Astr. Ap., 137, 343.

Schwering, P.B.W., 1988, Ph.D. Thesis (Terrewacht Leiden).

Shapiro, P.R., and Field, G.B. 1976, Ap.J., 205, 762.

Singh, K.P., Garmire, G.P., Nousek, J.A. 1987, Ap. J., 312, 484.

Spitzer, L. 1956, Ap.J., 124, 20.

Wang, Q., Hamilton, T.T., and Helfand, D.J. 1989, Nature, 341, 309.

Wang, Q., Hamilton, T.T., Helfand, D.J. and Wu, X., 1990, Ap.J., in press.

Wang, Q. 1990, "An X-ray Image of the Large Magellanic Cloud and a Study of its Hot Interstellar Medium," Columbia University Dissertation.

Wang, Q., and Helfand, D.J. 1990, (these proceedings).

Wang, Q. and Helfand, D.J. 1990a, Ap.J. (submitted).

Wang, Q. and Helfand, D.J. 1990b, Ap.J. (submitted).

Wang, Q. and Helfand, D.J. 1990c, Ap.J. (submitted).

Wu, X., Hamilton, T.T., Helfand, D.J., and Wang, Q. 1990, Ap.J. (submitted).

York, D.G. 1974, Ap. J. 193, L127. 